\title{
AGB Stars and the Observed Abundance of Neon in Planetary Nebulae
}

\author{
A. I. Karakas ${ }^{1,2}$ and J. C. Lattanzio ${ }^{1}$ \\ ${ }^{1}$ Centre for Stellar and Planetary Astrophysics, School of Mathematical Sciences, \\ Monash University, Melbourne 3800, Australia \\ john.lattanzio@sci.monash.edu.au \\ ${ }^{2}$ Institute for Computational Astrophysics, Department of Astronomy \& Physics, \\ Saint Mary's University, Halifax, Canada \\ akarakas@ap.stmarys.ca
}

Received 2003 November 3, accepted 2003 December 5

\begin{abstract}
Asymptotic giant branch stars are expected to produce ${ }^{22} \mathrm{Ne}$ through the combined $\mathrm{H}$ and $\mathrm{He}$ burning that operates during their thermally pulsing evolution. However, observationally there is a fairly tight correlation between the $\mathrm{O}$ and $\mathrm{Ne}$ abundances as measured in planetary nebulae in various populations. In this paper we use recent detailed stellar evolutionary calculations for compositions appropriate to the Galaxy and the Large Magellanic Cloud, in an attempt to determine if the models are consistent with the observed abundances. We show that there is only a narrow range in stellar mass, about 2 to $4 \mathrm{M}_{\odot}$ (lower for lower $[\mathrm{Fe} / \mathrm{H}])$ where ${ }^{22} \mathrm{Ne}$ is produced in sufficient quantities to affect the total observed elemental neon abundance, which is mostly ${ }^{20} \mathrm{Ne}$. The models appear to be consistent with the observations, but a more thorough analysis is required.
\end{abstract}

Keywords: stars: AGB and post-AGB — stars: abundances — stars: interiors — stars: low mass — ISM: abundances - planetary nebulae: general - neon

\section{Introduction}

After the asymptotic giant branch (AGB) phase is terminated, low to intermediate mass stars evolve through the planetary nebula $(\mathrm{PN})$ phase before finally ending their lives as white dwarfs. The gaseous nebula that makes up a PN is the remnant of the deep convective envelope that once surrounded the core, which is now exposed as the central star of the PN. Thus the abundances of the nebula should reveal information about the chemical processing that took place during the AGB and, more precisely, information about the final thermal pulses. The spectra of planetary nebulae (PNe) are usually composite, a mixture from the nebula and the illuminating central star. Many PNe are sufficiently large or bright that the nebular spectrum can be isolated (Kaler 1997). In the following discussion we do not examine the 'very late thermal pulses' that seem to be required to convert a H-rich central star to a He-rich central star; instead we refer the reader to Blöcker (2001).

One can accurately observe helium and neon abundances in PNe, unlike in a typical stellar photosphere (Kaler 1978; Henry 1989; Dopita et al. 1997; Stanghellini et al. 2000). This is because a strong ultraviolet flux from the central star of the PN has ionised the surrounding gas. The ionised gas recombines at excited levels, with the decay producing observable emission lines. Some of the strongest emission lines observed in PNe are the so-called forbidden lines, such as those produced by doubly ionised oxygen, $\mathrm{O}^{2+}$ [O III] (Kaler 1997). Other forbidden emission lines are [Ne III] and [O II], observed in the ultraviolet, and [N $\mathrm{II}]$ and $[\mathrm{S} \mathrm{II}]$, which are observed at longer wavelengths. The forbidden emission lines form in PNe (and diffuse nebulae) because of the low densities, where collisional de-excitation is so slow that a photon is emitted after a time of the order of seconds (Pagel 1997). The $\mathrm{C}, \mathrm{N}$ and $\mathrm{O}$ abundances can also be accurately measured, as well as a host of rare elements including $\mathrm{S}, \mathrm{Cl}$ and Ar (Dopita et al. 1997) and also elements affected by s-processing, such as Ge (Sterling, Dinerstein, \& Bowers 2002) and Zn (Dinerstein \& Geballe 2001).

\subsection{The Observations}

The observations of neon in PNe can be used as a powerful tool to constrain models of AGB stars. A correlation is observed to exist between the $\mathrm{Ne} / \mathrm{H}$ and $\mathrm{O} / \mathrm{H}$ abundance in PNe in the Galaxy, LMC, SMC and M31, within a small spread (Henry 1989). Dopita et al. (1997) find the same result for the Magellanic Clouds, and conclude that there is no sign of the dredge-up of ${ }^{22} \mathrm{Ne}$. Stasinska, Richer, \& McCall (1998) extend the work of Henry (1989) to a larger range of PNe, including a small sample in M32, and also observe a tight correlation between the neon and oxygen abundance. It seems that all $\mathrm{PNe}$, regardless of location, seem to have the same $\mathrm{Ne} / \mathrm{O}$ ratio (by mass) of $\sim 0.20$; it is assumed therefore that the Ne, Ar, and $\mathrm{S}$ abundances are unaltered by stellar evolution (Henry 1989; Stasinska et al. 1998), and that these elements are produced in the same relative proportions in massive stars (Stasinska et al. 1998). Do these observations present a problem for standard models?

Before we answer that question, let us summarise the chemical signature expected from the operation of 
the third dredge-up (TDU) in thermally-pulsing AGB (TP-AGB) models. Repeated dredge-up following thermal pulses will mix to the surface the products of both hydrogen and helium burning. From partial He-burning the surface is enriched in ${ }^{4} \mathrm{He}$ and ${ }^{12} \mathrm{C}$; some ${ }^{16} \mathrm{O}$ is also produced by $\alpha$-capture onto ${ }^{12} \mathrm{C}$ but the amount created is small in standard models without diffusive convective overshoot (see Herwig 2000). The ${ }^{12} \mathrm{C}$ mixed to the surface (as well as any present initially in the star) is then subjected to burning by the hydrogen shell, and the operation of the $\mathrm{CN}$ cycle transmutes this ${ }^{12} \mathrm{C}$ into ${ }^{14} \mathrm{~N}$. Once this material is exposed to helium burning, two $\alpha$-captures may transform each ${ }^{14} \mathrm{~N}$ nucleus into a ${ }^{22} \mathrm{Ne}$ nucleus. At the next dredge-up episode this ${ }^{22} \mathrm{Ne}$ is mixed to the surface, so we expect some enhancement in ${ }^{22} \mathrm{Ne}$ as well. For the intermediate mass stars (between say 4 and $7 \mathrm{M}_{\odot}$ ) the helium burning will include ${ }^{22} \mathrm{Ne}(\alpha, n)^{25} \mathrm{Mg}$ and ${ }^{22} \mathrm{Ne}(\alpha, \gamma)^{26} \mathrm{Mg}$. Hence the dredged-up material is enriched in these heavy Mg isotopes (Karakas \& Lattanzio 2003). As an additional complication, for masses above about $4 \mathrm{M}_{\odot}$ (depending on composition) the bottom of the convective envelope dips into the top of the hydrogen burning shell, in a phenomenon known as hot bottom burning (HBB). This further exposes the envelope to hydrogen burning during the interpulse phase, burning $\mathrm{CNO}$ into $\mathrm{N}$, ${ }^{22} \mathrm{Ne}$ into ${ }^{23} \mathrm{Na}$ and $\mathrm{Mg}$ isotopes into $\mathrm{Al}$ isotopes.

How does all this affect the Ne/O ratio? The Ne measured in $\mathrm{PNe}$ is the elemental neon abundance, and hence is a sum of all the isotopes. The ${ }^{20} \mathrm{Ne}$ isotope is the most abundant, and it is not altered significantly by $\mathrm{H}$ or He-burning during the AGB. Therefore only when the ${ }^{22} \mathrm{Ne}$ abundance exceeds or is equal to the ${ }^{20} \mathrm{Ne}$ abundance should we expect an enhancement in the $\mathrm{Ne} / \mathrm{O}$ ratio.

The linear relationship between the $\mathrm{Ne}$ and $\mathrm{O}$ abundance in PNe from many different locations is illustrated in the upper panel of Figure 1. In this diagram we include the observed abundances for over $300 \mathrm{PNe}$ in the Galaxy, LMC, SMC and M31, taken from Stasinska et al. (1998, hereafter S98), Henry (1989, hereafter H89) and 19 Galactic PN from S.R. Pottasch (2003, private communication). Results for 10 of those $19 \mathrm{PN}$ are given in Marigo et al. (2003), two in Pottasch et al. (2003a), one in Pottasch et al. (2003b) and one in Bernard-Salas et al. (2003). We shall collectively call these data P2003. The Galactic data from S98 are taken from the bulge of the Milky Way, but Exter et al. (2001) note that there is no difference between the average abundances and the abundance relationships for $\mathrm{PNe}$ in the bulge and disk. Even though there is some dispersion in the data, the correlation between the $\mathrm{Ne} / \mathrm{H}$ and $\mathrm{O} / \mathrm{H}$ ratios is clearly demonstrated. The errors in this diagram are probably of the order of 0.2 dex for $\mathrm{Ne} / \mathrm{H}$ and $\mathrm{O} / \mathrm{H}$ (Stanghellini et al. 2000). Stanghellini et al. (2000) examined the systematic differences between PNe abundances from different sources and found that the differences are within the errors for all elements but nitrogen. The elemental abundances are derived by using an ionisation correction factor, which can be large for low ionisation
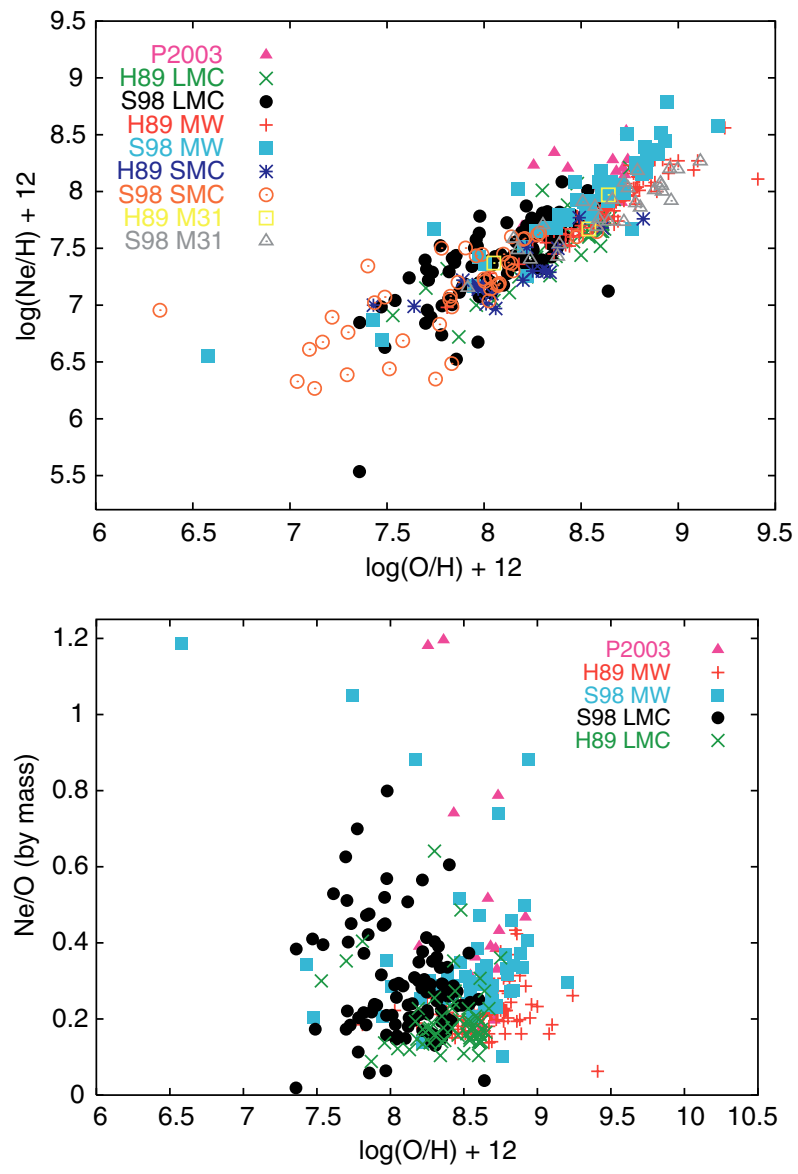

Figure 1 Upper panel: Observed $\mathrm{Ne} / \mathrm{H}$ versus $\mathrm{O} / \mathrm{H}$ for $\mathrm{PNe}$ in the Galaxy LMC, SMC and M31, taken from Stasinska et al. (1998), Henry (1989) and S.R. Pottasch (2003, private communication). Lower panel: The Ne/O ratio (by mass) for the Galaxy and LMC from the same data set.

PNe (Alexander \& Balick 1997), giving artificially high neon and sulphur abundances. Stanghellini et al. (2000) comment that there is no correlation between high neon abundances and low ionisation systems, and most of the systems analysed have high ionisation.

The problem of using incorrect or high ionisation correction factors can be done away with by using far infrared ISO (Infrared Space Observatory) observations along with optical data to calculate PN abundances (S.R. Pottasch 2003 , private communication). These abundances will be much more accurate than just using visual data alone because infrared data do not depend on electron temperature, the number of ions observed may double, which reduces the uncertainty in the ionisation correction factor substantially and extinction corrections may be determined in an independent way (Pottasch, Beintema, \& Feibelman 2000). Indeed, for the cases of neon, oxygen and argon, ionisation correction factors are no longer needed (S.R. Pottasch 2003, private communication). When the results calculated from ISO data are compared to other recent abundance determinations, large errors (sometimes of factors two to three) are found (see Pottasch et al. 2000 for an example). 
We will restrict the following discussion to $\mathrm{PNe}$ in the Galaxy and LMC, as most of the data are for these two systems. In the lower panel of Figure 1 we show the $\mathrm{Ne} / \mathrm{O}$ ratio (by mass) as a function of the oxygen abundance for $\mathrm{PNe}$ in the Galaxy and LMC using the same data set as plotted in the upper panel. In this diagram, we include the observed abundances for just over $200 \mathrm{PNe}$, most of which have a Ne/O ratio of about 0.2 . Kaler (1978) finds no variation to the $\mathrm{Ne} / \mathrm{O}$ ratio for Galactic $\mathrm{PNe}$, and Henry (1989) extends that observation to other systems. Even so, the spread in the data from S98 is rather large, even allowing for large error bars of \pm 0.2 to 0.3 dex. Many of the LMC and bulge PNe observed by $\mathrm{S} 98$ have Ne/O ratios far greater than 0.2 , and the largest ratio in the sample is about 1.2. The Ne/O ratio for the bulge PNe also seems to have a noticeable positive gradient, where PNe with the lowest $\log (\mathrm{O} / \mathrm{H})+12$ values are observed to have slightly lower $\mathrm{Ne} / \mathrm{O}$ ratios. This effect is probably connected to the initial chemical composition of the stars that produce these $\mathrm{PNe}$, and thus is related to galactic chemical evolution effects.

We conclude from Figure 1 that some of the dispersion is real, and that the $\mathrm{PNe}$ with the largest $\mathrm{Ne} / \mathrm{O}$ ratios are either enriched in neon or depleted in oxygen. If it is oxygen depletion, then this is unlikely to have taken place during the AGB. Although HBB can indeed deplete oxygen, it is not particularly efficient, reducing the ${ }^{16} \mathrm{O}$ abundance by only 0.3 dex in the most extreme case we calculated $(M=6, Z=0.008)$. Hence the most plausible scenario is that the star was born with a low oxygen abundance. There is, however, evidence that PNe can become enriched in neon. Comparing the properties of elliptical and bipolar PNe, Corradi \& Schwarz (1995) conclude that bipolar PNe are produced from more massive progenitors than the other PNe classes, and that bipolar PNe have overabundances of helium, nitrogen and neon. Stanghellini et al. (2000) provide evidence that the morphology of PNe is related to the abundances, and find that all asymmetric $\mathrm{PNe}$, which supposedly originate from a more massive progenitor (Corradi \& Schwarz 1995), have higher neon abundances on average than the symmetric PNe. It is unclear from the discussion in Stanghellini et al. (2000) if this effect is caused by chemical evolution, or an actual $\mathrm{Ne}$ enhancement from internal nucleosynthesis.

S.R. Pottasch (2003, private communication) recently completed an abundance analysis for 19 Galactic PN. These results are shown in Figure 1 as the solid pink triangles. A clear correlation between $\mathrm{Ne} / \mathrm{H}$ and $\mathrm{O} / \mathrm{H}$ is shown in the top panel of Figure 1, although four of the 19 PN sit in the upper left part of the diagram, and the same is true for the lower panel of Figure 1, where we plot the $\mathrm{Ne} / \mathrm{O}$ ratio (by mass). This indicates that either neon has been produced in these four nebulae or that oxygen has been destroyed. We note that a plot of $\mathrm{Ne} / \mathrm{H}$ versus $\mathrm{Ar} / \mathrm{H}$ would show a very clear straight line for all nebulae, with less than a $20 \%$ deviation (which can be the measurement error). This means that either argon is being produced in exactly the same quantity as neon, or that neither is being produced and the four low values of oxygen are due to oxygen destruction. That oxygen destruction by HBB is hard to achieve, at least in our models, might present something of a problem for the standard models.

\section{Stellar Models}

Models were calculated with the Mount Stromlo Stellar Structure code, updated to include the OPAL opacities of Iglesias \& Rogers (1996). We discuss the details of the code used and the initial abundances in Frost \& Lattanzio (1996) and Karakas, Lattanzio, \& Pols (2002). The reader is also referred to Wood \& Zarro (1981) and Lattanzio (1986). Mass loss was included using the prescription of Vassiliadis \& Wood (1993) but without the modification for $M$ greater than $2.5 \mathrm{M}_{\odot}$. We calculated model sequences for three different initial compositions: $Z=0.02,0.008$, and 0.004 over a range in mass $1 \leqslant M_{0}\left(\mathrm{M}_{\odot}\right) \leqslant 6$ where $M_{0}$ is the initial stellar mass.

We use the standard mixing-length theory for convection, with a mixing-length parameter $\alpha=l / H_{P}=1.75$. We do not include convective overshoot in our models even though there is evidence that it may be important on the main sequence for intermediate-mass stars (Chiosi et al. 1989; Schaller et al. 1992). The latest models suggest that the amount of overshooting required is small (Barmina, Girardi, \& Chiosi 2002), and for simplicity we have ignored overshoot in the models to be presented. We included semiconvection during core He-burning using the prescription described in Lattanzio (1986). We find the convective boundary at the base of the outer envelope by searching for a neutral border to the convective zone, in the manner described in Frost \& Lattanzio (1996) and Karakas et al. (2002). We note that this method does increase the efficiency of the TDU. Nevertheless, for lowmass models we do not find any dredge-up for the $Z=0.02$ models with $M \leqslant 2.0 \mathrm{M}_{\odot}$. Reaction rates used in the evolution code were taken mostly from Caughlan \& Fowler (1988), but with updates included in the nucleosynthesis calculations (see below).

We performed detailed nucleosynthesis calculations separately using a post-processing nucleosynthesis code which includes time-dependent mixing in all convective zones. The nucleosynthesis code takes as input from the evolution code structural variables such as temperature and density as a function of time and internal mass and calculates its own mass mesh, time step and abundances. The initial $\mathrm{H},{ }^{4} \mathrm{He}$ and $\mathrm{CNO}$ abundances used in the nucleosynthesis code are also taken from the zero age main sequence evolution model, detailed in the next section. In the nucleosynthesis network there are 74 nuclear species: from neutrons and protons up to sulphur there are 59 nuclei, with another 14 iron group species to allow for neutron capture on iron seed nuclei. There is also an additional 'particle' $g$ which has the function of counting the number of neutron captures occurring beyond ${ }^{61} \mathrm{Ni}$. The reaction network is terminated by a neutron capture on ${ }^{61} \mathrm{Ni}$ followed by an ad hoc decay, producing the particle represented by the symbol $g:{ }^{61} \mathrm{Ni}(n, \gamma){ }^{62} \mathrm{Ni} \rightarrow{ }^{61} \mathrm{Ni}+g$. 
Following the method of Jorissen \& Arnould (1989), neutron captures on the missing nuclides are modelled by giving the ${ }^{34} \mathrm{~S}(n, \gamma){ }^{35} \mathrm{~S}$ and ${ }^{61} \mathrm{Ni}(n, \gamma){ }^{62} \mathrm{Ni}$ reactions some averaged cross section values in order to represent all nuclei from ${ }^{34} \mathrm{~S}$ to ${ }^{54} \mathrm{Fe}$ and from ${ }^{61} \mathrm{Ni}$ to ${ }^{209} \mathrm{Bi}$ respectively. Apart from $\mathrm{H},{ }^{4} \mathrm{He},{ }^{12} \mathrm{C},{ }^{14} \mathrm{~N}$ and ${ }^{16} \mathrm{O}$, all other species begin with either solar or scaled solar values (see below).

The bulk of the 506 reaction rates are from the Reaclib Data Tables, based on the 1991 updated version of the compilation by Thielemann, Arnould, \& Truran (1991). We include recent reaction rates for $\alpha$, proton and neutron capture reactions when available, as detailed in Lugaro (1998, 2001).

\subsection{Initial Abundances}

Initial abundances for the solar composition $(Z=0.02)$ models were taken from Grevesse, Noels, \& Sauval (1992), and the Large Magellanic Cloud compositions $(Z=0.008)$ and Small Magellanic Cloud compositions ( $Z=0.004)$ were from Russell \& Dopita (1992). The evolutionary calculations require only six species: $\mathrm{H},{ }^{3} \mathrm{He}$, ${ }^{4} \mathrm{He},{ }^{12} \mathrm{C},{ }^{14} \mathrm{~N}$ and ${ }^{16} \mathrm{O}$, and we present these initial abundances (in mass fractions) in Table 1 . Note that we set ${ }^{3} \mathrm{He}=0$ initially. All other species are taken from the above references, where possible. For species not given in Russell \& Dopita (1992) we use scaled solar values, including initial isotopic ratios. Hence, in particular, the $\mathrm{Ne}$ and $\mathrm{Mg}$ isotopic ratios take their solar values in all our calculations.

In Table 2 we present the abundances (in mass fraction) of the $\mathrm{Ne}, \mathrm{Na}$ and $\mathrm{Mg}$ isotopes and $\mathrm{Ne} / \mathrm{O}$ (by mass) used in

Table 1. Initial compositions (in mass fractions) used for the stellar models

\begin{tabular}{lccc}
\hline & $\begin{array}{c}Z=0.02 \\
\text { Solar }\end{array}$ & $\begin{array}{c}Z=0.008 \\
\text { LMC }\end{array}$ & $\begin{array}{c}Z=0.004 \\
\text { SMC }\end{array}$ \\
\hline $\mathrm{H}$ & 0.6872 & 0.7369 & 0.7484 \\
${ }^{4} \mathrm{He}$ & 0.2928 & 0.2551 & 0.2476 \\
${ }^{12} \mathrm{C}$ & $3.4080 \times 10^{-3}$ & $9.6959 \times 10^{-4}$ & $4.8229 \times 10^{-4}$ \\
${ }^{14} \mathrm{~N}$ & $1.0542 \times 10^{-3}$ & $1.4240 \times 10^{-4}$ & $4.4695 \times 10^{-5}$ \\
${ }^{16} \mathrm{O}$ & $9.6000 \times 10^{-3}$ & $2.6395 \times 10^{-3}$ & $1.2830 \times 10^{-3}$ \\
Other $Z$ & $5.9378 \times 10^{-3}$ & $4.2484 \times 10^{-3}$ & $2.1899 \times 10^{-3}$ \\
\hline
\end{tabular}

Table 2. Initial abundances (in mass fraction) of $\mathrm{Na}$ and the $\mathrm{Ne}$ and $\mathrm{Mg}$ isotopes in the $Z=0.02$ and $Z=0.008$ models; also the $\mathrm{Ne} / \mathrm{O}$ ratio (by mass)

\begin{tabular}{lcc}
\hline Species & $Z=0.02$ & $Z=0.008$ \\
\hline${ }^{20} \mathrm{Ne}$ & $1.808 \times 10^{-3}$ & $5.215 \times 10^{-4}$ \\
${ }^{21} \mathrm{Ne}$ & $4.620 \times 10^{-6}$ & $1.333 \times 10^{-6}$ \\
${ }^{22} \mathrm{Ne}$ & $1.452 \times 10^{-4}$ & $4.188 \times 10^{-5}$ \\
${ }^{23} \mathrm{Na}$ & $3.818 \times 10^{-5}$ & $2.396 \times 10^{-4}$ \\
${ }^{24} \mathrm{Mg}$ & $5.904 \times 10^{-4}$ & $4.129 \times 10^{-4}$ \\
${ }^{25} \mathrm{Mg}$ & $7.750 \times 10^{-5}$ & $5.420 \times 10^{-5}$ \\
${ }^{26} \mathrm{Mg}$ & $8.892 \times 10^{-5}$ & $6.219 \times 10^{-5}$ \\
$\mathrm{Ne} / \mathrm{O}$ & 0.203 & 0.212 \\
\hline
\end{tabular}

the $Z=0.02$ and $Z=0.008$ models. The initial $\mathrm{Ne} / \mathrm{O} \sim$ 0.2 is consistent with abundances observed in both PNe and HII regions in the LMC by Vermeij \& van der Hulst (2002) and in spiral galaxies by van Zee et al. (1998). Note the abundance of $\mathrm{Na}$ in the $Z=0.008$ models is larger than the solar value and about 15 times larger than the scaled solar value.

\subsection{Model Results}

The neon isotope ${ }^{22} \mathrm{Ne}$ is efficiently produced in the He-burning shell of AGB stars, from the ${ }^{14} \mathrm{~N}$ ashes of previous $\mathrm{CN}$ cycling. In Figure 2 we show the ${ }^{22} \mathrm{Ne}$ mass fraction in the intershell of all the stellar models we compute. The ${ }^{22} \mathrm{Ne}$ abundances are taken from the intershell at the end of the last calculated thermal pulse, and therefore reflect the abundances that are mixed into the envelope by the subsequent TDU episode. The $M \sim 3 \mathrm{M}_{\odot}$ models have the largest ${ }^{22} \mathrm{Ne}$ intershell mass fractions. This conclusion does not depend strongly on the metallicity, but there is a shift toward lower mass as the initial metallicity is decreased. The reason that the peak ${ }^{22} \mathrm{Ne}$ intershell abundance does not depend on the metallicity is because of the third dredge-up, which increases the ${ }^{12} \mathrm{C}$ abundance in the envelope. Hence, there is more ${ }^{12} \mathrm{C}$ available in the $\mathrm{H}$ shell to convert to ${ }^{14} \mathrm{~N}$ by $\mathrm{CN}$ cycling. As discussed above, each ${ }^{14} \mathrm{~N}$ nucleus may capture two $\alpha$ particles to become $\mathrm{a}^{22} \mathrm{Ne}$ nucleus. Since the $3 \mathrm{M}_{\odot}$ models also have efficient TDU, where $\lambda \gtrsim 0.7$, we should expect these models to produce the most ${ }^{22} \mathrm{Ne}$, a result which is almost independent of the initial metallicity. In the more massive models, ${ }^{22} \mathrm{Ne}$ is efficiently destroyed by $\alpha$-capture and at lower masses there is little or no dredge-up, so the ${ }^{22} \mathrm{Ne}$ is not brought to the surface.

We can compare the predicted intershell values with the surface abundance observations of PG 1159-type objects, which are thought to be in transition from central star of PNe to white dwarf (Napiwotzki 1998). These objects are quite rare, with only about two dozen known, and their

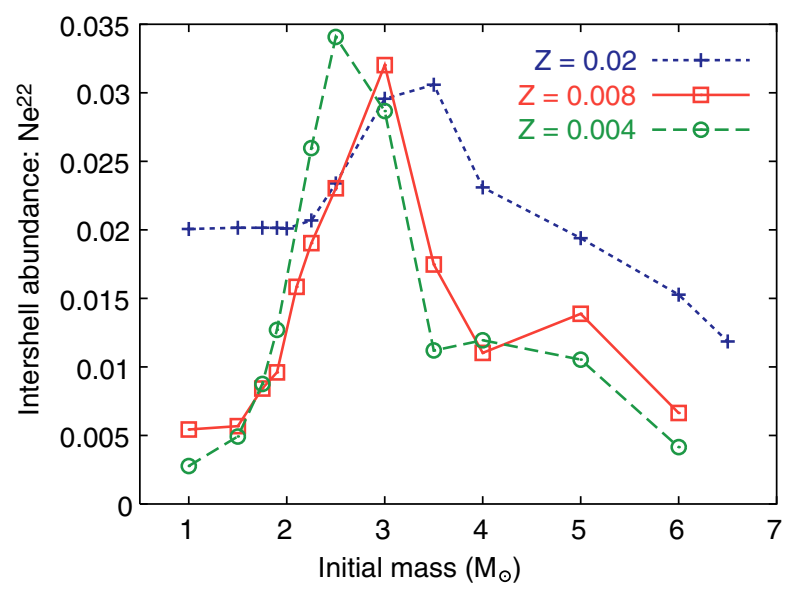

Figure 2 The ${ }^{22} \mathrm{Ne}$ mass fraction in the intershell as a function of the initial mass. The ${ }^{22} \mathrm{Ne}$ abundances are taken from the last calculated thermal pulse, just after the convective pocket has dissipated. 

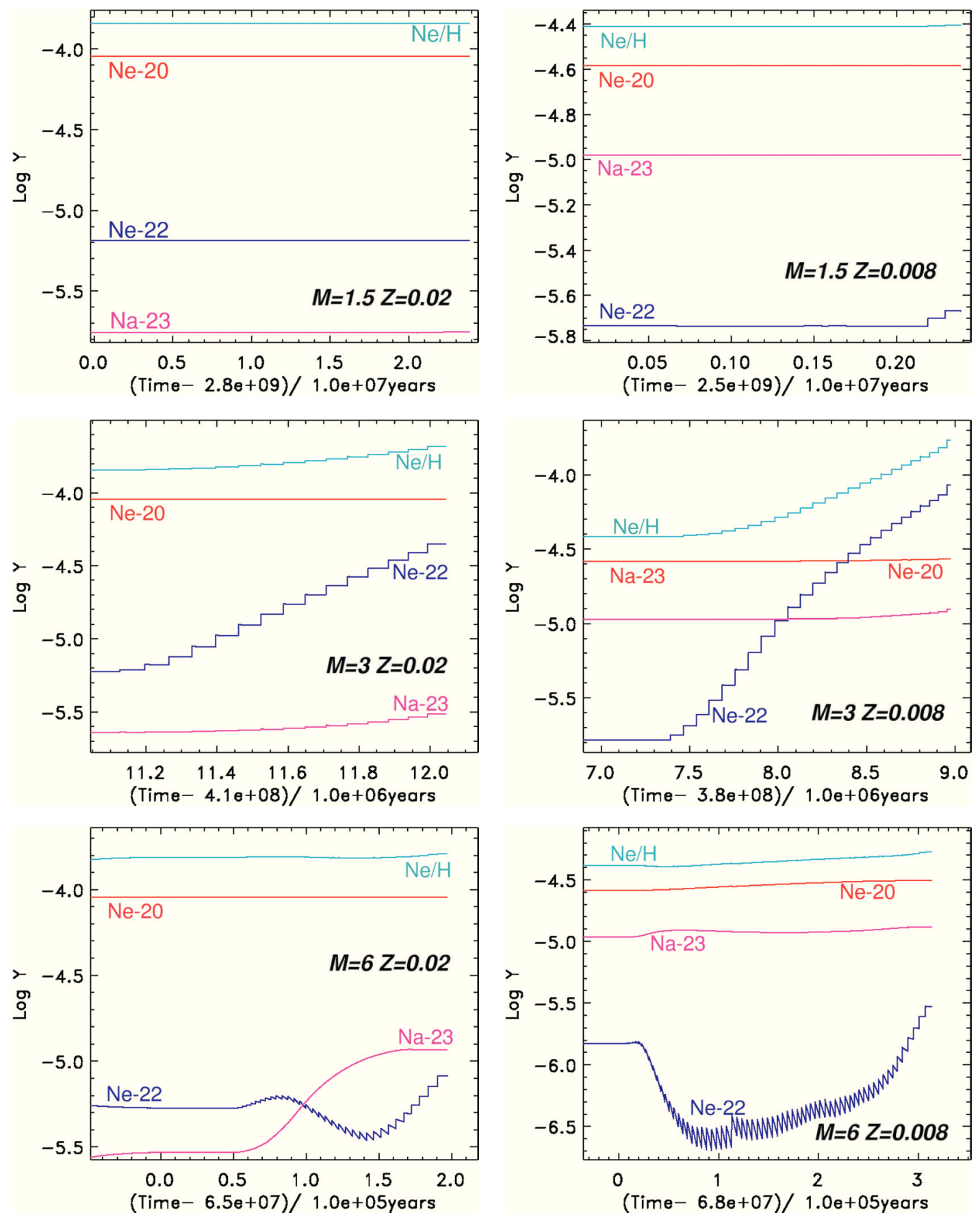

Figure 3 The abundances of ${ }^{20} \mathrm{Ne},{ }^{22} \mathrm{Ne}$ and ${ }^{23} \mathrm{Na}$ as well as the $\mathrm{Ne} / \mathrm{H}$ ratio during the $\mathrm{TP}-\mathrm{AGB}$ phase for the $1.5,3 \mathrm{M}_{\odot}$ and $6 \mathrm{M}_{\odot}, Z=0.02$ (left) and $Z=0.008$ (right) models.

atmospheres are mostly composed of helium, carbon and oxygen (Werner \& Rauch 1994). Spectroscopic observations of neon reveal mass fractions of about $2 \%$ to $5 \%$ (Werner \& Wolff 1999), consistent with the abundances ${ }^{1}$

${ }^{1}$ Observations of the PG 1159 central stars reveal oxygen mass fractions as high as $20 \%$ (Werner \& Rauch 1994), clearly at odds with standard stellar models. To reproduce the large oxygen abundances observed, extra-mixing processes are required to bring ${ }^{16} \mathrm{O}$ from the $\mathrm{CO}$ core into the intershell region. The diffusive convective overshooting models of Herwig (2000) have intershell abundances that are consistent with the abundance patterns observed in PG 1159 central stars. The degenerate thermal pulses found by Frost, Lattanzio, \& Wood (1998) could also have in Figure 2. Thus the models are predicting the right amount of neon in the intershell; it is the amount of dredgeup that determines the neon enrichment in the gaseous nebulae.

In Figure 3 we show the surface abundance of ${ }^{20} \mathrm{Ne}$, ${ }^{22} \mathrm{Ne}$ and $\mathrm{Ne} / \mathrm{H}$ as a function of time for the 1.5, 3 and $6 \mathrm{M}_{\odot}$ models. The left-hand column presents results for

a similar effect. In this case, the third dredge-up following the degenerate pulse can mix material from the $\mathrm{CO}$ core into the envelope, thus enhancing the envelope in ${ }^{16} \mathrm{O}$. Whilst this idea is certainly promising, a quantitative study is required. 
the $Z=0.02$ models and the right-hand column presents results for the $Z=0.008$ models. The $Z=0.02$ models are assumed to be representative of Galactic PNe and the $Z=0.008$ models are assumed to be representative of LMC PNe. From Figure 3 we see that the lower mass models $\left(M<2.5 \mathrm{M}_{\odot}\right)$ experience very little or no dredge-up, so the change to the elemental $\mathrm{Ne} / \mathrm{H}$ abundance is negligible. In the most massive models, $\alpha$-capture destroys some of the ${ }^{22} \mathrm{Ne}$ in the intershell, plus ${ }^{22} \mathrm{Ne}$ is destroyed by the $\mathrm{Ne}-\mathrm{Na}$ chain to produce ${ }^{23} \mathrm{Na}$ by HBB. However in these models, the abundance of ${ }^{20} \mathrm{Ne}$ is slightly enhanced from the destruction of ${ }^{23} \mathrm{Na}$. Overall, the change to the $\mathrm{Ne} / \mathrm{H}$ ratio is small. In the $6 \mathrm{M}_{\odot}, Z=0.02$ model the change to the $\mathrm{Ne} / \mathrm{H}$ ratio is much less than $0.1 \mathrm{dex}$ and in the $6 \mathrm{M}_{\odot}, Z=0.008$ model, the $\mathrm{Ne} / \mathrm{H}$ ratio increases by about 0.1 dex. These results imply that there will be little change to the neon abundances in PNe originating from low-mass progenitors, and only a small increase in the neon abundance in PNe from the most massive progenitors. An increase of 0.1 dex, as predicted by the models, is probably not observable.

Because the $M \sim 3 \mathrm{M}_{\odot}$ models produce the most ${ }^{22} \mathrm{Ne}$ during a thermal pulse (see Figure 2), we would expect considerable ${ }^{22} \mathrm{Ne}$ enrichment in these models. In Figure 3 we see this to be the case, with the largest ${ }^{22} \mathrm{Ne}$ enhancements occurring in the $3 \mathrm{M}_{\odot}$ models, regardless of the metallicity. This large increase actually occurs over a small mass range, from $2.5 \mathrm{M}_{\odot}$ to $3.5 \mathrm{M}_{\odot}$, for both the solar and LMC metallicity models. In the case of the $3 \mathrm{M}_{\odot}$, $Z=0.02$ model, the change to the $\mathrm{Ne} / \mathrm{H}$ ratio during the TP-AGB is about 0.2 dex. Since the errors are also about 0.2 dex (Stanghellini et al. 2000), this is probably not a significant increase. The change to the $\mathrm{Ne} / \mathrm{H}$ ratio for the $3 \mathrm{M}_{\odot}, Z=0.008$ model is about $0.6 \mathrm{dex}$, and should be observable. We note for comparison that the change to the $\mathrm{Ne} / \mathrm{H}$ ratio for the $2.5 \mathrm{M}_{\odot}$ and $3.5 \mathrm{M}_{\odot}, Z=0.008$ models is about 0.4 dex and 0.3 dex respectively. This analysis also highlights another important aspect of the models, that the most metal-poor models have the largest increase in the surface $\mathrm{Ne} / \mathrm{H}$ ratio. Most $\mathrm{PNe}$ are observed to have $\mathrm{Ne} / \mathrm{O}$ ratios of about 0.2 , with a linear relationship between the $\mathrm{Ne} / \mathrm{H}$ and $\mathrm{O} / \mathrm{H}$ abundances. Can these observations help constrain the stellar models? In other words, is the TDU too efficient in the low-metallicity models with $M \sim 3 \mathrm{M}_{\odot}$ ? To try to answer this question, we now compare the stellar models directly to the observations.

\subsection{Confrontation between Models and Data}

Before we go on, we should point out that a direct comparison between the data and our models is not exactly valid. A detailed comparison would require unfolding the effects of known metallicity gradients observed in both the Galaxy and the LMC, as well as chemical evolution effects. Rather we will compare with specific compositions. Nevertheless, we will still be able to say something about the $\mathrm{Ne}$ enhancement expected at a given metallicity, or $\log (\mathrm{O} / \mathrm{H})$ value.
The gaseous nebulae that comprise the $\mathrm{PNe}$ have masses of only a few $\times 0.1 \mathrm{M}_{\odot}$. Hence they should show the nucleosynthetic results of the last thermal pulse. For the purposes of comparison to the observations in Figure 1 we will focus on the surface abundance after the last dredge-up episode.

However, the stellar models presented in Figure 3 do not, in all cases, extend to the very end of the AGB. At the highest luminosities, there are often severe convergence problems which prevent us from calculating this very final stage. For the lower mass models the remaining envelope is small, usually less than $0.2 \mathrm{M}_{\odot}$ and hence less than the amount expected to be lost during the next interpulse period. But for the intermediate mass models there can be up to $\sim 1.5 \mathrm{M}_{\odot}$ of envelope mass remaining. For the intermediate masses, thermal pulses and the TDU may in principle continue, so we use an algorithm to simulate the evolution and enrichment of these few remaining thermal pulses. This is discussed in detail in Karakas \& Lattanzio (2003), but briefly we use the details of the nucleosynthesis and evolution at the last calculated pulse, together with the principles of synthetic evolutionary calculations, to estimate the effect of these few remaining pulses. We note that the efficiency of the third dredge-up, as measured through the dredge-up parameter $\lambda$, becomes a free parameter for these few remaining pulses. We do not know how dredge-up proceeds as the envelope mass decreases, but there is some evidence that the efficiency is decreased (Straniero et al. 1997; see also Karakas et al. 2002). Thus we calculate the remaining thermal pulses using three values of $\lambda$ : (1) $\lambda=\lambda_{\text {last }}$, (2) $\lambda=0.3$ and (3) $\lambda=0.0$, where $\lambda_{\text {last }}$ is the efficiency of the third dredge-up evaluated from the last two thermal pulses (see Karakas \& Lattanzio 2003). In the following discussion, we only discuss cases (1) and (3), which are the two extremes.

From the upper panel of Figure 4 we see that the $Z=0.02$ models with $\lambda=0$ (blue stars) fit well within the range of the Galactic PNe observed by H89 and P2003, and the $Z=0.02$ models with $\lambda=\lambda_{\text {last }}$ sit well within the range of $\mathrm{Ne} / \mathrm{O}$ occupied by the bulge PNe. From the lower panel of Figure 4 we see that most of the $Z=0.008$ points fit within the $\mathrm{Ne} / \mathrm{O}$ range occupied by the observations, with the exception of two points. Both of these points are for the $3 \mathrm{M}_{\odot}, Z=0.008$ model. The blue star at $\mathrm{Ne} / \mathrm{O} \sim 0.9$ represents the $3 \mathrm{M}_{\odot}$ model with $\lambda=0$ for the remaining dredge-up episodes and the pink box at $\mathrm{Ne} / \mathrm{O} \sim 1.2$ represents the case where $\lambda=\lambda_{\text {last }}=0.86$. The point at $\mathrm{Ne} / \mathrm{O} \sim$ 0.9 fits within the errors, if we assume the errors are at least of the order of \pm 0.2 , but the point at $\mathrm{Ne} / \mathrm{O} \sim 1.2$ does not. $\mathrm{S} 98$ reported $\mathrm{PNe}$ in the bulge with $\mathrm{Ne} / \mathrm{O}$ ratios of at least 1.0 at the $\log (\mathrm{O} / \mathrm{H})+12$ ratios consistent with the LMC. If the models are correctly predicting that a narrow mass range of stars produce enough ${ }^{22} \mathrm{Ne}$ to increase the $\mathrm{Ne} / \mathrm{O}$ ratios, then perhaps we have not observed enough LMC $\mathrm{PNe}$ to find the few with large $\mathrm{Ne} / \mathrm{O}$ ratios? Since there are over 150 LMC PNe shown in Figure 4, the question then becomes one of completeness of the sample: do the data adequately cover this narrow mass range? Alternatively, 

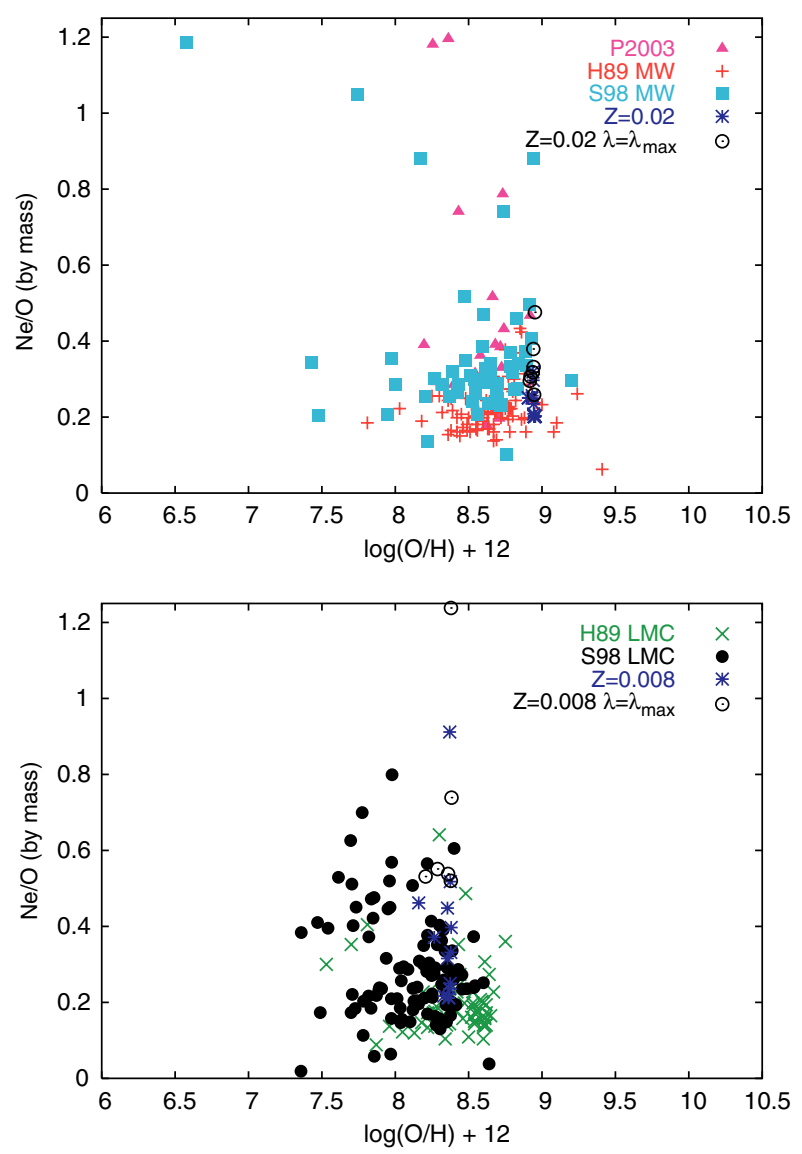

Figure 4 Upper panel: The Ne/O ratio for the Galactic PNe, with the results for the $Z=0.02$ models included. Lower panel: Same, but for the LMC and over-plotted with the results for the $Z=0.008$ models.

the $M \sim 3 \mathrm{M}_{\odot}$ models may be producing too much ${ }^{22} \mathrm{Ne}$. This could certainly be the case for the $3 \mathrm{M}_{\odot}, Z=0.008$ model with $\lambda=\lambda_{\text {last }}$, but the same case with $\lambda=0$ sits within the errors of the observed points. Maybe these observed neon abundances can be used as a constraint on the cessation of the third dredge-up in the AGB models? Indeed, one would not expect the deep dredge-up to continue right to the end of the AGB and the fact that the data argue for $\lambda=0$ for the last pulse is perfectly reasonable and consistent both with our expectations and the models.

We mention for comparison that the models of Herwig (2000), with diffusive convective overshoot, also experience very efficient TDU ( $\lambda \gtrsim 1)$, but because these models also produce more ${ }^{16} \mathrm{O}$ in the intershell than our models it is unclear how the $\mathrm{Ne} / \mathrm{O}$ ratio will change during the TP-AGB phase.

In summary, we conclude that the $Z=0.02$ models fit well within the range of neon abundances observed in Galactic PNe. Most of the $Z=0.008$ model points also fall within the range of neon abundances observed in LMC $\mathrm{PNe}$, with the possible exception of the $3 \mathrm{M}_{\odot}, Z=0.008$ model (if the deep dredge-up continues to the very end of the AGB). That we find potential disagreement for a very narrow range of mass is both rewarding and curious. The simplest explanation is that the TDU efficiency decreases with vanishing envelope mass, as expected on many grounds.

\section{Conclusion}

We return now to our original question: are the neon abundances observed in PNe a problem for standard stellar models? The answer to that question appears to be 'no'. Certainly there is no disagreement for the Galactic objects, and if the dredge-up efficiency decreases near the end of the AGB then the LMC models are also consistent with the observations, although there is a possibility that the models predict too much neon in a very narrow mass range.

In this discussion, we have concentrated on the observations of neon in PNe but there are also abundances for $\mathrm{He}, \mathrm{C}, \mathrm{N}$ and $\mathrm{O}$ that should also be considered. Dopita et al. (1997) state that there is clear evidence for the dredge-up of carbon, consistent with the efficient operation of the TDU. There are also PNe with large $\mathrm{He} / \mathrm{H}$ and high N/O ratios, perhaps consistent with the operation of $\mathrm{HBB}$ (Dopita et al. 1997). But Dopita et al. (1997) also mention that agreement between the observations and theory would be better reached if $\mathrm{HBB}$ occurred at $M \gtrsim 2 \mathrm{M}_{\odot}$, perhaps indicating that some form of deep mixing is yet again required. A detailed comparison between our results and various PNe abundances will be the subject of a subsequent paper.

\section{Acknowledgments}

AIK wishes to acknowledge the Astronomical Society of Australia, Monash Cluster Computing Laboratory, and the Victorian Partnership for Advanced Computing for funding, and the Australian Partnership for Advanced Computing supercomputer facility for computer time. This research was supported by the Australian Research Council. We also thank Mike Dopita for useful discussions, and Stuart Pottasch for useful discussions and for providing results ahead of publication.

\section{References}

Alexander, J., \& Balick, B. 1997, AJ, 114, 713

Barmina, R., Girardi, L., \& Chiosi, C. 2002, A\&A, 385, 487

Bernard-Salas, J., Pottasch, S. R., Beintema, D. A., \& Feibelman, W. A. 2003, A\&A, 406, 165

Blöcker, T. 2001, Ap\&SS, 275, 1

Caughlan, G. R., \& Fowler, W. A. 1988, ADNDT, 40, 283

Chiosi, C., Bertelli, G., Meylan, G., \& Ortolani, S. 1989, A\&A, 219,167

Corradi, R. L. M., \& Schwarz, H. E. 1995, A\&A, 293, 871

Dinerstein, H. L., \& Geballe, T. R. 2001, ApJ, 562, 515

Dopita, M., et al. 1997, ApJ, 474, 188

Exter, K., Barlow, M. J., Walton, N. A., \& Clegg, R. E. S. 2001, Ap\&SS, 277, 199

Frost, C. A., \& Lattanzio, J. C. 1996, ApJ, 344, L25

Frost, C. A., Lattanzio, J. C., \& Wood, P. R. 1998, ApJ, 500, 355

Grevesse, N., Noels, A., \& Sauval, A. J. 1992, in Proceedings of the First SOHO Workshop: Coronal Streams, Coronal Loops, and Coronal and Solar Wind Composition (ESA SP-348), 305

Henry, R. C. B. 1989, MNRAS, 241, 453 (H89)

Herwig, F. 2000, A\&A, 360, 952 
Iglesias, C. A., \& Rogers, F. J. 1996, ApJ, 464, 943

Jorissen, A., \& Arnould, M. 1989, A\&A, 221, 161

Kaler, J. B. 1978, ApJ, 255, 527

Kaler, J. B. 1997, Stars and their Spectra (Cambridge: Cambridge University Press)

Karakas, A. I., \& Lattanzio, J. C. 2003, PASA, 20, 279

Karakas, A. I., Lattanzio, J. C., \& Pols, O. R. 2002, PASA, 19,515

Lattanzio, J. C. 1986, ApJ, 311, 708

Lugaro, M. A. 1998, in Proc. of the Fifth International Symposium on Nuclei in the Cosmos, eds. N. Prantzos \& S. Harissopulos (France: Editions Frontiéres), 501

Lugaro, M. A. 2001, PhD Thesis, Monash University

Marigo, P., Bernard-Salas, J., Pottasch, S. R., Tielens, A. G. G. M., \& Wesselius, P. R. 2003, A\&A, 409, 619

Napiwotzki, R. 1998, in Reviews in Modern Astronomy 11: Stars and Galaxies, ed. R. E. Schielicke (Germany: Astronomische Gesellschaft), 3

Pagel, B. E. J. 1997, Nucleosynthesis and Chemical Evolution of Galaxies (Cambridge: Cambridge University Press)

Pottasch, S. R., Beintema, D. A., \& Feibelman, W. A. 2000, A\&A, 363, 767

Pottasch, S. R., Bernard-Salas, J., Beintema, D. A., \& Feibelman, W. A. 2003a, A\&A, 409, 599
Pottasch, S. R., Hyung, S., Aller, L. H., Beintema, D. A., Bernard-Salas, J., Feibelman, W. A., \& Klökner, H.-R. 2003b, A\&A, 401, 205

Russell, S. C., \& Dopita, M. A. 1992, ApJ, 384, 508

Schaller, G., Schaerer, D., Meynet, G., \& Maeder, A. 1992, A\&SS, 96, 269

Stanghellini, L., Shaw, R. A., Balick, B., \& Blades, J. C. 2000, ApJ, 534, L167

Stasinska, G., Richer, M. G., \& McCall, M. L. 1998, A\&A, 336, 667 (S98)

Sterling, N. C., Dinerstein, H. L., \& Bowers, C. W. 2002, ApJ, 578, L55

Straniero, O., Chieffi, A., Limongi, M., Busso, M., Gallino, R., \& Arlandini, C. 1997, ApJ, 478, 332

Thielemann, F.-K., Arnould, M., \& Truran, J. W. 1991, in Advances of Nuclear Astrophysics, ed. E. Vangioni-Flam et al. (France: Editions Frontiéres), 525

Werner, K., \& Rauch, T. 1994, A\&A, 284, L5

Werner, K., \& Wolff, B. 1999, A\&A, 347, L9

Wood, P. R., \& Zarro, D. M. 1981, ApJ, 248, 311

van Zee, L., Salzer, J. J., Haynes, M. P., O’Donoghue, A. A., \& Balonek, T. J. 1998, AJ, 116, 2805

Vassiliadis, E., \& Wood, P. R. 1993, ApJ, 413, 641

Vermeij, R., \& van der Hulst, J.M. 2002, A\&A, 391, 1081 